\title{
Modeling Renewable Penetration Using a Network Economic Model
}

\author{
A. Lamont
}

March 6, 2001

U.S. Department of Energy

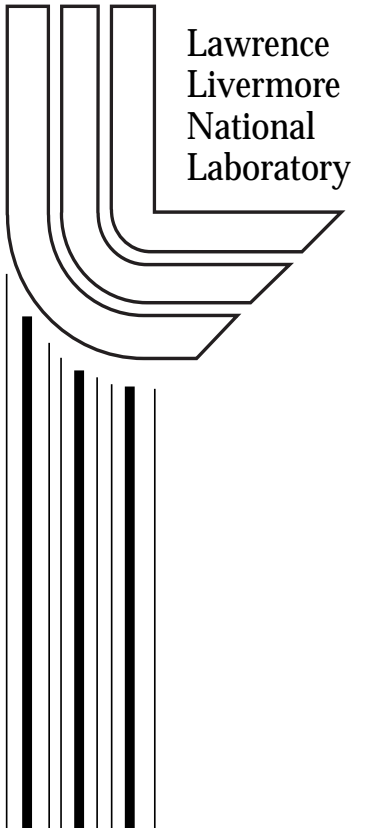




\section{DISCLAIMER}

This document was prepared as an account of work sponsored by an agency of the United States Government. Neither the United States Government nor the University of California nor any of their employees, makes any warranty, express or implied, or assumes any legal liability or responsibility for the accuracy, completeness, or usefulness of any information, apparatus, product, or process disclosed, or represents that its use would not infringe privately owned rights. Reference herein to any specific commercial product, process, or service by trade name, trademark, manufacturer, or otherwise, does not necessarily constitute or imply its endorsement, recommendation, or favoring by the United States Government or the University of California. The views and opinions of authors expressed herein do not necessarily state or reflect those of the United States Government or the University of California, and shall not be used for advertising or product endorsement purposes.

This work was performed under the auspices of the U. S. Department of Energy by the University of California, Lawrence Livermore National Laboratory under Contract No. W-7405-Eng-48.

This report has been reproduced directly from the best available copy.

Available to DOE and DOE contractors from the

Office of Scientific and Technical Information

P.O. Box 62, Oak Ridge, TN 37831

Prices available from (423) 576-8401

http://apollo.osti.gov/bridge/

Available to the public from the National Technical Information Service

U.S. Department of Commerce 5285 Port Royal Rd., Springfield, VA 22161

http://www.ntis.gov/

OR

Lawrence Livermore National Laboratory Technical Information Department's Digital Library http://www.llnl.gov/tid/Library.html 


\title{
Modeling Renewable Penetration Using a Network Economic Model
}

\author{
Alan Lamont \\ Lawrence Livermore National Laboratory \\ Livermore, CA, USA \\ Lamont1@IInl.gov
}

\begin{abstract}
This paper evaluates the accuracy of a network economic modeling approach in designing energy systems having renewable and conventional generators. The network approach models the system as a network of processes such as demands, generators, markets, and resources. The model reaches a solution by exchanging prices and quantity information between the nodes of the system. This formulation is very flexible and takes very little time to build and modify models. This paper reports an experiment designing a system with photovoltaic and base and peak fossil generators. The level of PV penetration as a function of its price and the capacities of the fossil generators were determined using the network approach and using an exact, analytic approach. It is found that the two methods agree very closely in terms of the optimal capacities and are nearly identical in terms of annual system costs.
\end{abstract}

\section{Introduction}

Network economic modeling has been used at Lawrence Livermore National Laboratory to optimize the design and operation of energy system that include conventional and renewable energy technologies as well as storage technologies. Network models are built as a network of nodes, each node representing a process in the energy system. Using an iterative algorithm, nodes exchange price and quantity information, adjusting their capacities and outputs in response to this information. Eventually the system reaches an equilibrium. Under the right conditions, this equilibrium is a cost minimizing solution for both the configuration and the operation of the system.

Network models have the advantage that they are easy to set up and modify. Systems having thousands, or tens of thousands, of equations and constraints can be set up in a few minutes. This approach 
can also handle many non-linear behaviors of components when needed. This paper examines the accuracy of the network modeling approach in designing energy systems that include renewables.

Here we develop the design of a photovoltaic /fossil energy system using a network modeling system. This particular problem provides a good test of the network approach in that the model must a) select the correct level of photovoltaic (PV) penetration, and b) size the capacities of the fossil generators for a given level of $\mathrm{PV}$ penetration. This problem can be solved analytically to determine the theoretically optimal configuration and the minimum costs. The results from the network model can be directly compared to the theoretical results for evaluation.

These results provide a clearer picture of the penetration of the PV as a function of its price as well as an evaluation of the ability of network models to determine an optimal configuration for the system

\section{Background to network modeling approach}

The network approach was developed as a way to organize large scale models of the energy system into modular structures that are easier to construct, modify, and analyze. It was originally used in the SRI-Gulf model of the energy system (Cazalet). The software architecture of that model, however, was difficult to modify. Subsequently, two systems were developed to make it easier to build and modify energy models. One of these is the Livermore Economic Modeling System (LEMS) developed at Lawrence Livermore National Laboratory (Rousseau), and the other is the Generalized Equilibrium Modeling System (GEMS) developed by Decision Focus Inc. (Decision Focus Inc.) Both of these systems are built around a library of standard modules that represent a variety of processes. In the 1990's a second system, the META•Net economic modeling system (Lamont), was developed based on object oriented programming to simplify the development and modification of the software.

The systems described above are structured for solving long term problems: they deal with periods that are several years long and within a single model run a node might add capacity in earlier periods and then retire the capacity in later periods. For our current work, the modeling system has been adapted to short term modeling. In these examples the model horizon is one year divided into 8760 one hour periods. One of the primary differences between the short-term and long-term formulations is that the node capacities in a short-term model are fixed over the model horizon. The capacities are adjusted between iterations. But, during a 
single iteration the capacity is constant over all periods. Making this adaptation required the reprogramming of a few of the node models in the library.

Hogan and Weyant discuss both the value this approach and its theoretical basis. They point out that because of the size and complexity of many energy models, they are often difficult to develop, understand, and use. To alleviate this it is important that models have the following characteristics:

- a consistent underlying theory,

- a model structure that corresponds to the natural organization of the data and system,

- a modular design,

- decentralized implementation, and

- efficient computation.

These characteristics are met through the network approach to modeling: The nodes are small modular models of particular processes in the real world. The nodes exchange well defined, standard information about quantities and prices. Therefore, the structure of the system corresponds to the natural organization of the data and system. The modules, and the well defined protocols for communication, lead to a modular structure and make it possible to decentralize the development of the various pieces of the model. The network approach does not, in and of itself, lend itself to efficient computation. However, when considering the total effort in building, maintaining, and modifying a large model, it is expected that the network approach would lead to significant overall savings.

Much of the Hogan and Weyant paper is devoted to developing the underlying theory of network modeling. Essentially, they show that the network approach can be derived from, and is equivalent to, a standard optimization formulation for economic models. They show that this problem can be reformulated to decompose it into a set of smaller optimization problems corresponding to consumers and processes (e.g. producers). The nodes of the model execute these smaller optimization problems to arrive at an overall optimum for the entire system. 


\section{Computational approach used in network modeling}

This section gives an overview of the overall scheme of the calculations in a model and introduces the key computations in the different types of nodes. Figure 1 shows the network used for these analyses and can be used to illustrate the discussion below.

\section{Up and down passes}

There are two passes in the network algorithm. In the "down pass", quantities demanded are passed down from the demand nodes through the network to the resource nodes. During the "up pass" prices are passed from the resource nodes up to the demand nodes.

At the start of the down pass the demand node determines the quantity it requires at each period and passes it down to the market node (a circle in the diagram). The market node must efficiently allocate that demand to each of its suppliers. It uses market share function based on prices of the suppliers-suppliers with higher prices receive smaller shares and those with lower prices receive larger shares.

When the market node passes the quantities required down to the conversion nodes (squares), those nodes determine the amount of their inputs that they require in order to meet the demand. In the cases modeled here this is based simply on an input/output coefficient for the process. More complex nodes can also have non-linear relationships between input and output such as the case where the efficiency of a process decreases as the load on it is increased.

Finally, the quantities are passed down to the resource nodes (diamonds). At this point, the "up pass" starts. In this simple model, the resource nodes simply determine a price for the fuel they provide. In these examples, natural gas is provided at a uniform price over all hours. The "Sun" resource is essentially free. In more complex cases the resource nodes may represent exhaustible resources whose prices rise as the resource is depleted. In general, the prices charged by the resource nodes are the marginal costs of supplying additional increments of resource.

These prices are passed up to the conversion nodes. Given the prices of their inputs, they determine the price they must charge. This is discussed below. They pass these prices to the market node which takes a quantity weighted average price and passes that on up to the demand node. 
In the general case, the demand node can be price sensitive and thus determine its demand based on the price. In this example however, the demand node has a fixed demand for each hour.

\section{Allocation of demands at market nodes}

The price sensitivity of the market is a parameter of the model. If the market sensitivity is 0 , all suppliers receive equal shares, regardless of price. With higher sensitivities, any supplier whose price is slightly above the lowest price will receive a nearly 0 share and the lowest price supplier will have a share of 1.0. However, as the demand to a supplier is increased, its price tends to increase, while the price tends to decrease as the demand is decreased. With a very price sensitive market, this process ultimately has the effect of making allocations so that all of the prices are nearly equal. The equalization of marginal prices is the condition for minimizing the costs within the markets.

\section{Constraints}

The renewable technologies have constraints in each hour, reflecting the hourly availability of the resource and the capacity that has been set. Each of the dispatchable technologies also have constraints reflecting the actual capacity available. These constraints are enforced by computing shadow prices whenever the demand to a node exceeds its constraint. The market allocations are actually based on these shadow prices. The shadow prices are adjusted until the constraints are just met. To assist in computing the correct shadow prices, the nodes also compute the derivative of quantity demanded with respect to price. These derivatives are passed down along with the quantity information.

\section{Capacity modeling}

The model also optimizes the capacities of the conversion processes. The capacities are adjusted each iteration, but once they are set, they are constant for the entire year. During an iteration, the current capacity of a node constitutes a constraint on production. Shadow prices are generated to ensure that this constraint is met. The shadow prices can also be used to measure the marginal value of additional capacity at the node. The node adjusts its capacity until the marginal value of additional capacity is equal to the marginal cost of additional capacity. Note that we can also place maximum constraints on the capacity for any node. 


\section{Description of the experiment}

The experiment develops a configuration and operating plan for a system consisting of a base fossil generator, a peak fossil generator, and a PV generator. Figure 1 illustrates the configuration. The electric demand is specified for each hour of the year (8760 hours). The loads used here are actual loads taken from the southwest United States. They are summer peaking due to air conditioning loads. For this experiment they have been scaled to a peak load of $1 \mathrm{~kW}$.

Two fossil generators are included in the system. One has the characteristics of a base load generator $(\$ 800 / \mathrm{kw}, 57 \%$ efficient) while the other is a peak generator ( $\$ 200 / \mathrm{kW}, 32 \%$ efficient). It is assumed that both are fueled by natural gas (at a price of $\$ 0.02 / \mathrm{kWh}$ ). The costs of the fossil generators are fixed for all the runs.

The PV generator output each hour is a function of the PV capacity and the hour-by-hour solar availability. The hourly solar data are taken from a recording station in central California.

In this experiment, the PV capital cost is varied and the optimal level of penetration is determined for each level of cost. As PV penetrates, the load duration curve for the two fossil generators

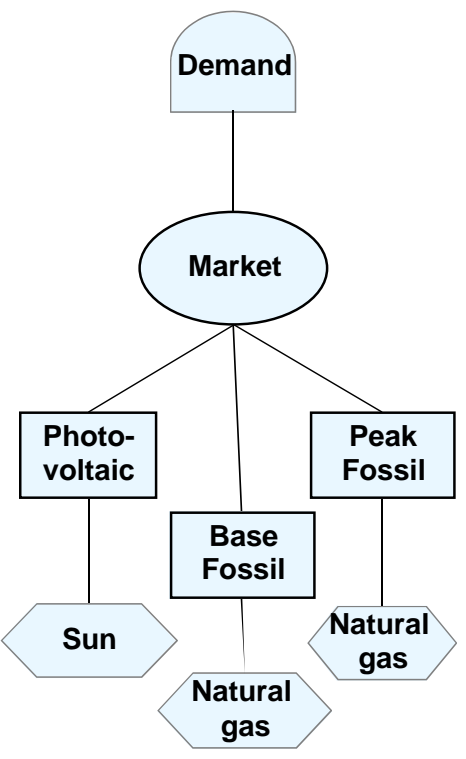
changes. This requires a change in the capacities of those generators. Our experiment, therefore, determines the changes in the PV and fossil capacities as a Figure 1: Model structure for experiment function of the PV cost. In addition, the total annual costs of the systems is determined using a $6 \%$ discount rate.

Even this simple system has in the order of 40,000 constraints (equality and inequality). We have developed models have as many as 140,000 constraints using this approach.

For this simple system, we can analytically determine the exact optimal configuration and costs. We compare this analytic solution to that obtained by the network model. 


\section{Analytic solution}

In the analytic solution we postulate a level of penetration for the $\mathrm{PV}$ and determine the optimal configuration and system costs for the fossil generators, given the level of PV penetration. Based on this information, the PV cost that would justify the postulated level of generation is calculated. The analytic solution is computed in the following steps:

- Postulate an amount of PV capacity.

- For each hour, determine the amount of PV energy available (given the postulated capacity) and subtract it from the electric load.

- Construct the LDC of the remaining load.

- Based on the LDC and the capital and operating costs of the two fossil generators, determine their optimal capacities.

- For each hour, determine the amount of generation from the two fossil generators, assuming that the generator with the lowest operating cost is dispatched first.

- Compute the total capital and operating costs of the fossil generators.

At this point we have determined the configuration and costs of the fossil generation for the postulated PV penetration. The next steps determine the PV cost that would justify that penetration. The cost can be determined from the following logic: Adding an increment of PV capacity reduces the cost of the fossil generators by reducing the amount of generation required and, possibly, by reducing the capacity required.

Adding an increment of PV will be economically justified if the cost of the $P V$ is less than or equal to the reduction in cost of the fossil system. The value of $P V$ is therefore the derivative of the fossil system costs taken with respect to PV penetration. By making successive analyses at small increments of PV capacity, we can estimate the derivatives and compute the cost of PV that corresponds to each level of PV penetration.

\section{Network Solution}

To compute the network solution, the model was run with selected values of the PV cost. Each solution provides the capacities of the PV and the fossil generators. It also provides the hour-by-hour dispatch of the generators. Given the cost of fuel, efficiencies of the generators and 
the other operating costs, the total annual capital and operating costs were computed.

\section{Results}

First the capacities given by the two solutions are compared. As is discussed below, these are not exact, but are reasonably close. A more important measure is the cost of the two solutions. These are within a few percent of each other. Fundamentally this result indicates that the cost minimum for these systems is very flat: deviations from the optimal solution result in very small changes in cost.

The PV penetration is shown as a function of PV cost in Figure 2. The analytic solution indicates that there will be no penetration until the price of PV drops slightly below $\$ U S 1,900 / \mathrm{kWp}$. The network solution gives some penetration at costs somewhat above $\$ \cup S 2,000 / \mathrm{kWp}$. Between the costs of $\$ U S 1,800$ and $400 / \mathrm{kWp}$ the capacities of the two solutions correspond reasonably well

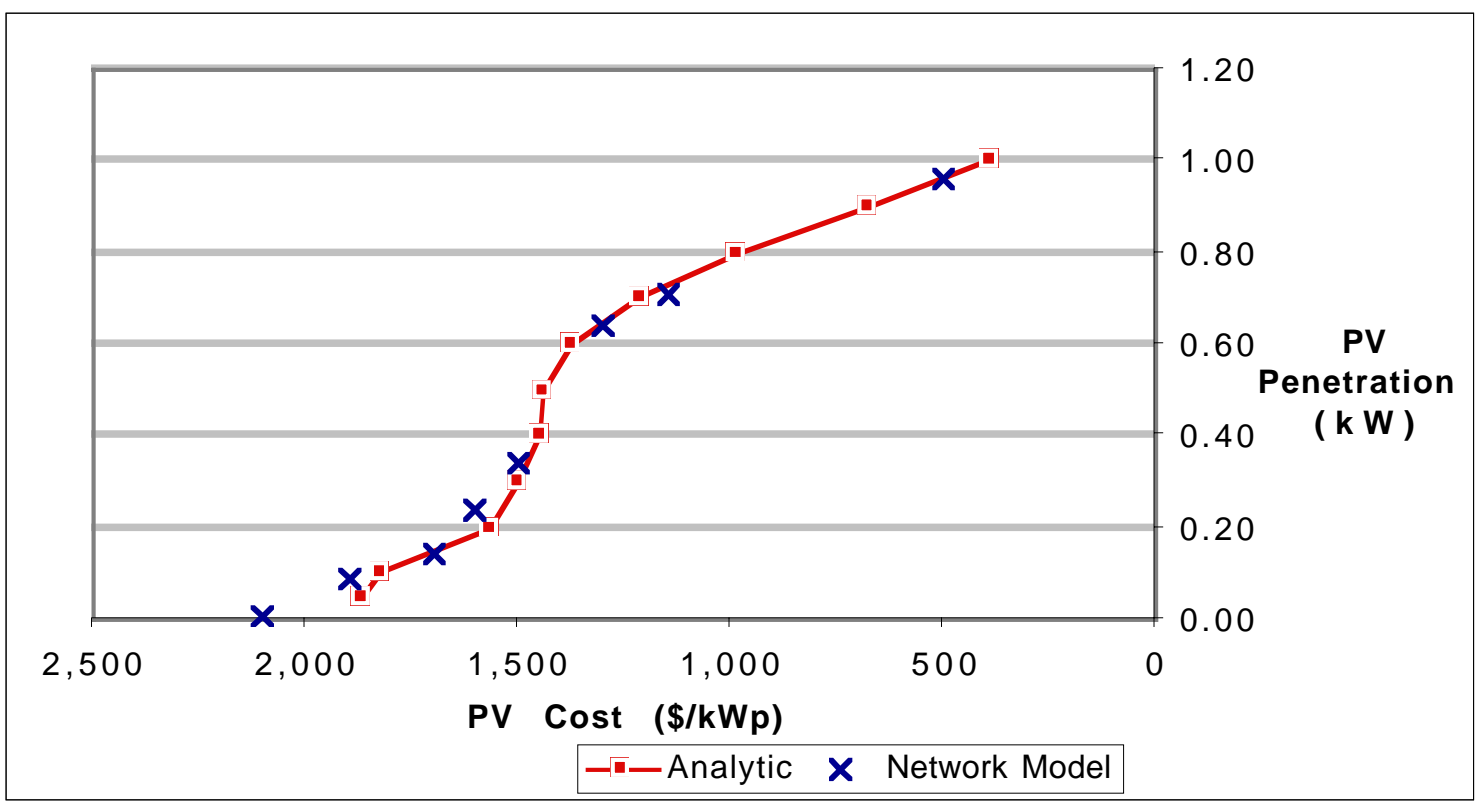

Figure 2: PV penetration as a function of the capital cost

The corresponding capacities for the two fossil generators are shown in Figure 3. When PV is initially introduced, it displaces both the peak and the base fossil capacity since the peak of the PV generation happens to coincide with the peak electric demand. Eventually PV no longer displaces capacity and only displaces generation. This changes the 
shape of the load duration curve for the fossil generators leading to an increase in the capacity of the peak generator.

The network model has a slight tendency to equalize the capacities of the fossil generators compared to the analytic solution. In these examples the price sensitivity of the market node was set to a high level so this tendency is minimized. With lower price sensitivities it is somewhat more apparent. However, even with lower price sensitivities and a somewhat non-optimal set of capacities for the fossil generators, the total annual system cost is still very close to the cost of the optimal system.

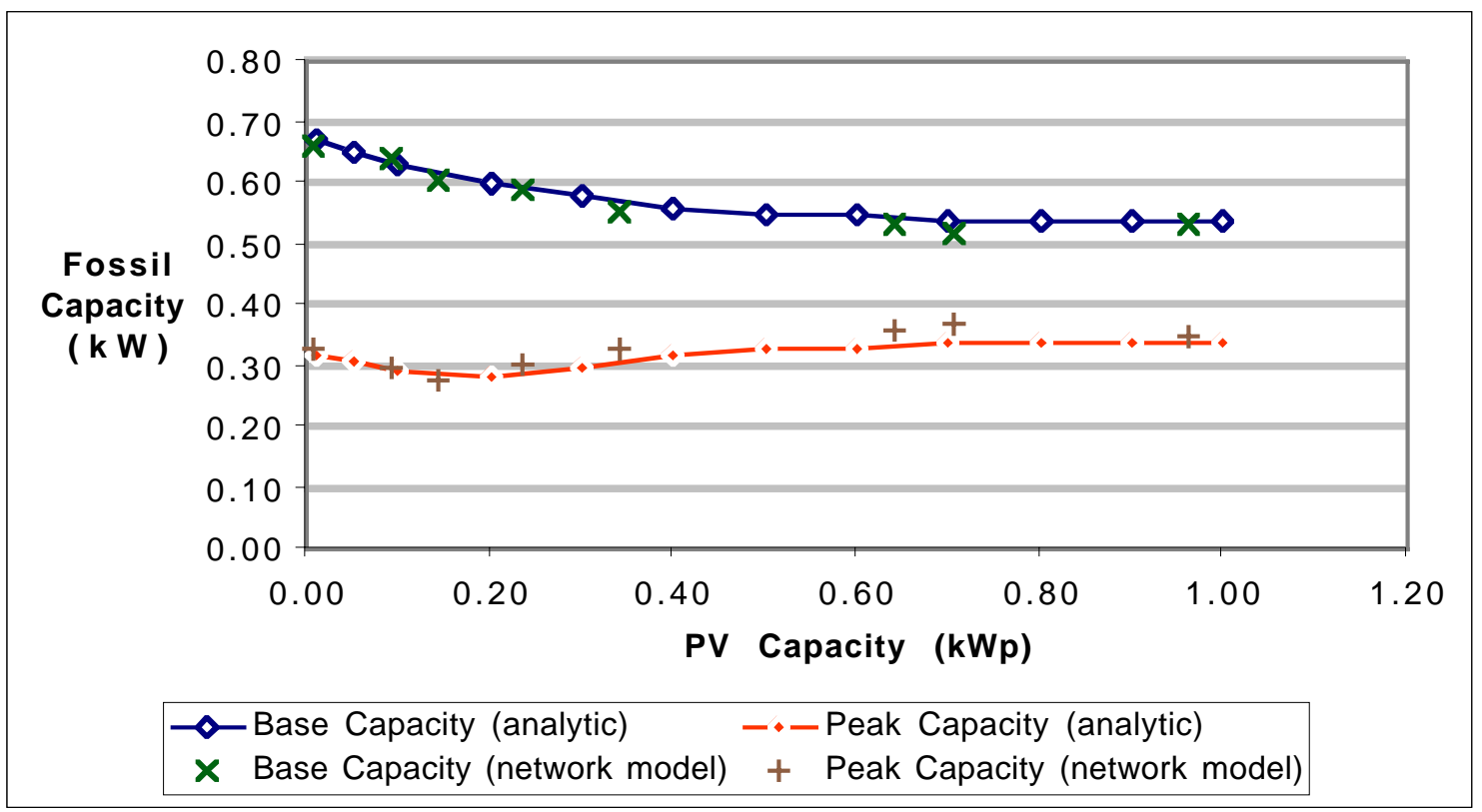

Figure 3: Capacities of fossil generators as a function of PV penetration

The costs of the systems are shown in Figure 4 as a function of PV penetration. These costs are reasonably close over the entire range. In these cost calculations, the cost of PV varies so that high PV capacity implies a low PV cost. It is interesting to note that the introduction of PV has very little effect on system cost until PV becomes very inexpensive. PV will, of course, have substantial benefits in terms of reducing emissions of all types. 


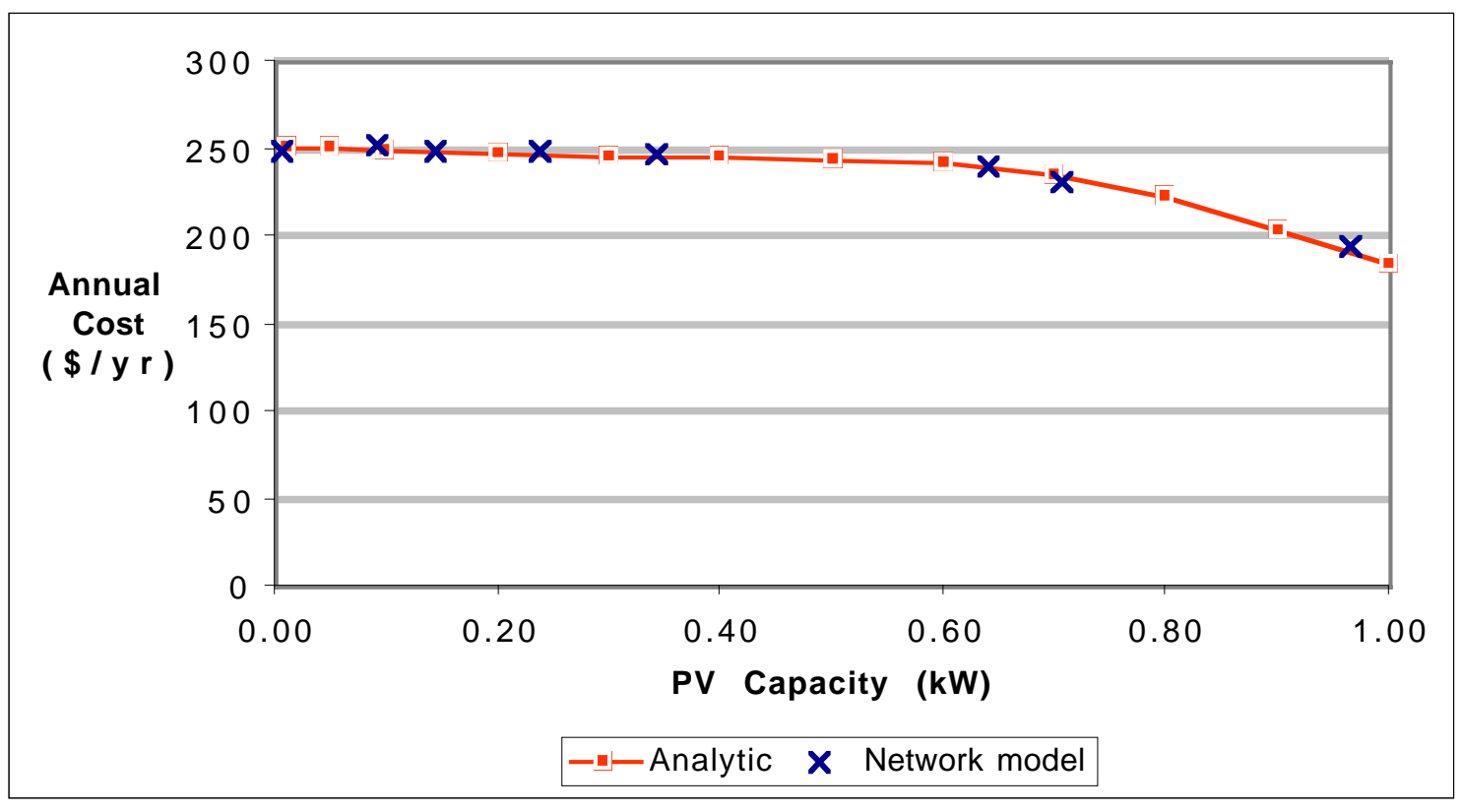

Figure 4: Total annual system costs as a function of PV capacity

\section{Conclusions about efficacy of network modeling approach}

The network modeling approach can be a valuable tool in designing systems with renewable, non-dispatchable generation. It does a good job in determining the correct level of renewable generators to be used and it makes the correct allocation of capacities between fossil generators based on the load duration curve. The costs of the systems determined using the network system are very close to the true optimal systems.

\section{References}

Cazalet, E. G., Generalized Equilibrium Modeling: The Methodology of the SRI-Gulf Energy Model, Final report prepared by Decision Focus Inc., Palo Alto, CA for the U. S. Federal Energy Administration, May 1977

Decision Focus Inc., The DFI Generalized Equilibrium Modeling System (GEMS), Decision Focus Inc., Mountain View CA, 1979

Hogan, William W. and John P. Weyant, (1982), "Combined Energy Models", in Advances in the Economics of Energy and Resources, Vol. 4, pages 117-150, JAI Press Inc. 
Lamont, A., User's guide to the META-Net Economic Modeling System; version 1.2, Lawrence Livermore National Laboratory, UCRL-ID-122511, 1994

Rousseau, W. F., S. S. Sussman, R. N. Castleton, and J. T. Rambo, Economic Models and Algorithms Used in the Livermore Economic Modeling System, Lawrence Livermore National Laboratory, Livermore CA, UCRL-52527, 1978 